\title{
An outreach intervention to implement evidence based practice in residential care: a randomized controlled trial [ISRCTN67855475] Maria Crotty*1, Craig Whitehead ${ }^{1}$, Debra Rowett ${ }^{2}$, Julie Halbert ${ }^{1}$, David Weller, Paul Finucane ${ }^{1}$ and Adrian Esterman ${ }^{3}$
}

\author{
Address: ${ }^{1}$ Department of Rehabilitation and Aged Care, Flinders University, Bedford Park Australia 5042, ${ }^{2}$ Drug and Therapeutic Information \\ Service, Repatriation General Hospital, Daw Park Australia 5041 and ${ }^{3}$ Clinical Epidemiology and Health Outcomes Unit, Flinders Medical Centre, \\ Bedford Park Australia 5042 \\ Email: Maria Crotty* - maria.crotty@flinders.edu.au; Craig Whitehead - craig.whitehead@rgh.sa.gov.au; \\ Debra Rowett - debra.rowett@rgh.sa.gov.au; Julie Halbert - julie.halbert@rgh.sa.gov.au; David Weller - david.weller@flinders.edu.au; \\ Paul Finucane - paul.finucane@rgh.sa.gov.au; Adrian Esterman - adrian.esterman@flinders.edu.au \\ * Corresponding author
}

Published: 06 April 2004

BMC Health Services Research 2004, 4:6

This article is available from: http://www.biomedcentral.com/I472-6963/4/6

(C) 2004 Crotty et al; licensee BioMed Central Ltd. This is an Open Access article: verbatim copying and redistribution of this article are permitted in all media for any purpose, provided this notice is preserved along with the article's original URL.

\begin{abstract}
Background: The aim of this project was to assess whether outreach visits would improve the implementation of evidence based clinical practice in the area of falls reduction and stroke prevention in a residential care setting.
\end{abstract}

Methods: Twenty facilities took part in a randomized controlled trial with a seven month follow-up period. Two outreach visits were delivered by a pharmacist. At the first a summary of the relevant evidence was provided and at the second detailed audit information was provided about fall rates, psychotropic drug prescribing and stroke risk reduction practices (BP monitoring, aspirin and warfarin use) for the facility relevant to the physician. The effect of the interventions was determined via pre- and post-intervention case note audit. Outcomes included change in percentage patients at risk of falling who fell in a three month period prior to follow-up and changes in use of psychotropic medications. Chi-square tests, independent samples t-test, and logistic regression were used in the analysis.

Results: Data were available from case notes at baseline $(n=897)$ and seven months follow-up $(n=902)$, 452 residential care staff were surveyed and $I 2 I$ physicians were involved with $6 I$ receiving outreach visits. Pre-and post-intervention data were available for 715 participants. There were no differences between the intervention and control groups for the three month fall rate. We were unable to detect statistically significant differences between groups for the psychotropic drug use of the patients before or after the intervention. The exception was significantly greater use of "as required" antipsychotics in the intervention group compared with the control group after the pharmacy intervention ( $R R=4.95 ; 95 \% \mathrm{Cl} 1.69-14.50$ ). There was no statistically significant difference between groups for the numbers of patients "at risk of stroke" on aspirin at follow-up.

Conclusions: While the strategy was well received by the physicians involved, there was no change in prescribing patterns. Patient care in residential settings is complex and involves contributions from the patient's physician, family and residential care staff. The project highlights challenges of delivering evidence based care in a setting in which there is a paucity of well controlled trial evidence but where significant health outcomes can be attained. 


\section{Background}

While some of the frailest members of our community live in residential care, their medical care is widely believed to be suboptimal. It is unclear to what extent the problems with treating this group is the result of lack of knowledge, discomfort about the risk/benefit profile of treatments in the very old or structural issues within residential care which inhibit clinical effectiveness. There is some evidence that improvements in prescribing can be achieved using outreach visits and educational strategies such as "academic detailing" with providers in residential care [1]. However there has been no examination of this approach when applied to the implementation of clinical guidelines within residential care.

Two key health areas for older adults in residential care are falls and stroke prevention. Falls and consequent injuries are a major public health problem in nursing home residents and herald a functional decline and loss of independence $[2,3]$. Achieving reductions in falls in residential care settings has proved difficult. While a trial in two nursing homes [4] and a trial using geriatric assessment [5] failed to reduce fall rates, a multidisciplinary consultative program targeting the $25 \%$ of nursing home patients at highest risk for falls was able to demonstrate a reduction in the number of falls [6]. The key elements of this program were an interdisciplinary approach with the appointment of a falls coordinator at each facility with responsibility for the implementation of the program.

Stroke is a leading cause of disability for elderly Australians [7]. While there is robust evidence that secondary prevention (identification and treatment of high risk groups) can improve outcomes with significant reductions in morbidity and death $[8,9]$, there is also evidence that primary care physicians are failing to apply this evidence in residential care settings. For example, only $40 \%$ of patients in one survey of secondary stroke prevention in nursing homes were shown to be adequately anticoagulated [10].

While outreach visit programs have been effective approaches to implementing evidence based practice in other primary care settings [11] they are yet to be evaluated in residential care. Therefore, the aim of the SAFIRE (Strokes And Falls In Residential care Evaluation) project was to examine the impact of an outreach visit intervention, targeting falls reduction and stroke prevention in a residential care setting. Our hypothesis was that prescribing patterns and risk factor modification would occur more frequently in those facilities who had received the outreach intervention than in those who had not.

\section{Methods}

The SAFIRE project was a randomised controlled trial involving a multifaceted intervention conducted in resi- dential care facilities in metropolitan Adelaide, South Australia. Ethics approval was granted by the Research and Ethics Committee, Repatriation General Hospital, Adelaide.

Facilities were chosen across the metropolitan health areas of Adelaide, South Australia. Each region was randomly allocated as intervention or control. The first facility to be included in the study was selected at random and matched to a facility in another region to avoid overlap of physicians. The remaining facilities were selected in the same manner until the cumulative number of residents met the estimated sample size required $(n=20$ facilities, 10 hostels - low level care and 10 nursing homes - high level care). The sample size was calculated assuming a $10 \%$ reduction in falls rate in the intervention group and estimated to be 348 . All randomisation was conducted using a computer-generated random allocation program by a person external to the project. Figure 1 presents a visual description of the study design. Informed consent was obtained from the resident or their next of kin in order to access case notes. A list of all primary care physicians attending each facility and the number of patients they treated was drawn up. Commencing with those physicians with the largest number of patients they were approached to take part in the study. Six physicians per facility (120 in total) were recruited for the study and all were only treating patients in either the intervention region or the control region.

All patients/carer of the participating residential care facilities were approached for consent to allow an audit of the clinical practice in each facility. Case notes were audited by nurses blinded to allocation for demographic information, diagnoses and stroke risk factors. Medication charts were reviewed for prescription and administration of any psychotropic and/or antihypertensive medication and use of aspirin or warfarin. Information was also collected on the number of falls and injurious falls in the last 12 months from incident report forms at each facility. Seven months after baseline measures were taken, the case note audit was repeated for all consenting residents.

Intervention physicians received two, 30 min detailing visits by a pharmacist in their surgeries. The key messages combined the evidence based guidelines on falls prevention $[12,13]$ with information from the case note audit and survey and included: the benefits of the use of aspirin in residents with increased stroke risk, the monitoring and treatment of hypertension, the benefits of the use of warfarin in residents with atrial fibrillation, and the risk of psychotropic drug use. There is little evidence specific to patients in residential care settings for the management of stroke risk factors. We adhered to guidelines for community based patients eg WHO guidelines for hypertension 
[14] and consulted with experts in the area. Physicians were also provided with detailed audit information on the facility where the physician worked including falls rates, psychotropic prescribing patterns, stroke risk reduction practices (BP monitoring, aspirin and warfarin use). The use of pharmacists to provide this information has been used in previous studies in this region and was familiar and acceptable to the physicians.

One nurse per facility was appointed as a link nurse. The link nurses underwent four two-hour sessions in change management, management of the behavioural symptoms of dementia, medication management and falls prevention techniques. In addition, a pharmacist visited each facility and spoke to staff on reducing the use of psychotropic medications.

The primary outcome for the project was the three month fall rate prior to the follow-up assessment. Secondary outcomes included 12 month fall rate, rate of injurous falls, recorded blood pressure readings from previous three months, prescription and administration of any psychotropic medication, prescription of aspirin or warfarin, or an antihypertensive, Modified Barthel Index - Activity of Daily Living (ADL) score [15], Nursing Home Life-Space Diameter - Mobility score [16] and The Nursing Home Problem Behaviour Scale [17].

To assess the reliability of the case note data extraction and reduce inter-observer error, five percent of records from the first audit had data extracted twice and 10\% of records were extracted twice from the second. Reliability (measured by Pearson's correlation coefficient for continuous variables and \% agreement for categorical variables) was $95 \%$ or greater on demographic and drug usage data.

Descriptive data are reported as percentages and means and standard deviation. Chi-squared tests were used for assessing differences in proportions between study groups, whereas Student's t tests were used to compare means. Relative risks for the effect of the intervention after adjusting for age, gender, level of care, dementia status, and baseline levels of the outcome measure were undertaken using generalized linear models. To account properly for the cluster randomisation design used, the facility was recognised as the primary sampling unit in all analyses. All statistical analyses were undertaken using the STATA statistical package [18].

\section{Results}

Twenty facilities were involved in the trial; 10 hostels (low level facilities) and 10 nursing homes (high level facilities). The total number of potential participants for the first case note audit was 1061 and of these, 897 (85\%) consented to participate. Next of kin consent was obtained in $50.4 \%$ (range $26-80 \%$ for facilities) of cases. At the second audit 1055 potential participants were available, 902 (85\%) consented to involvement with $46.7 \%$ requiring next of kin consent. Two hundred and two people $(22.5 \%)$ who participated at the first audit were not available at the second due to various reasons including death and moving facility. The attrition rate was as high as $37 \%$ in some facilities but there were no overall differences in attrition rate between the intervention and control facilities $(p=0.086)$. Those participants that were missing at the second audit were more likely to be male ( $\mathrm{p}$ $=<0.001)$ and be older $(\mathrm{p}=0.003)$ than participants available for follow-up at the second audit. There was no difference in the number of those diagnosed with dementia $(p=0.884)$, having antipsychotics or benzodiazepines administered regularly $(\mathrm{p}=0.40$ and $\mathrm{p}=0.346$ respectively), or having fallen in the previous three months ( $\mathrm{p}=$ 0.768 ). Of the 121 primary care physicians approached to participate, $98(81 \%)$ consented with 61 attending intervention and 37 attending control facilities, respectively. GPs in the control region had larger numbers of patients under their care. These physicians cared for $59 \%$ of the residential care residents whose notes were audited.

Table 1 describes the baseline characteristics of the residents. At baseline, residents from the intervention group were more likely to have resided at the facility for less than three months $(\mathrm{p}=0.04)$, were more likely to have a diagnosis of dementia $(\mathrm{p}=0.01)$ and were more likely to have documentation of not for resuscitation ( $p<0.001)$. There were no differences between groups for a diagnosis of depression, length of stay at facility or mean number of recent unplanned hospital visits. Residents in the intervention group facilities were more likely to be prescribed a psychotropic medication or benzodiazepine on an "as required" (PRN) basis than those in the control facilities $(p<0.05)$. Seventy-one percent were at risk of falls or stroke, $48 \%$ had fallen in the last 12 months and $23 \%$ of residents had had a stroke in the past. Sixty-seven percent were prescribed a psychotropic medication, with a higher rate in nursing homes compared with hostels $(74 \% \mathrm{v}$ $62 \%$ ). Overall $35 \%$ of residents were on aspirin. However, of those identified at high risk of stroke (prior stroke/TIA or with a known risk factor) only $45 \%$ were treated with aspirin. Less than half of the residents identified at risk of stroke (35\% in nursing homes and $43 \%$ in hostels) had a blood pressure measurement recorded in the prior three months.

Over the period of the intervention, the percentage of residents who reported one or more falls in the previous three months increased for both the intervention and control groups, however, there were no differences in the fall rate between groups (Table 2 ). 
Table I: Characteristics of residents at baseline $(n=715)$.

\begin{tabular}{lcc}
\hline Characteristics of residents at baseline & Control $(\mathbf{n}=\mathbf{3 3 4})$ & Intervention $(\mathbf{n}=\mathbf{3 8} \mathbf{I})$ \\
\hline Age $(\mathbf{y r}) *$ & $83.4 \pm 7.9$ & $84.7 \pm 7.7$ \\
Gender - Male (\%) & 18.0 & 14.5 \\
$\quad$ Female (\%) & 82.0 & 85.5 \\
Diagnosis of dementia in case notes n (\%) & $103(33.4)$ & $162(42.7)$ \\
Diagnosis of depression in case notes n (\%) & $64(19.2)$ & $67(17.8)$ \\
Length of Stay in residential care facility (days) & $40.1 \pm 39.5$ & $35.7 \pm 39.5$ \\
Length of Stay - less than 3 months (\%) & 5.4 & 9.4 \\
Length of Stay - less than I2 months (\%) & 24.4 & 29.7 \\
Documented "not for resuscitation" in case notes (\%) & 1.9 & 10.3 \\
Mean number of unplanned hospital visits in prior 3 months* & $0.08 \pm 0.38$ & $0.05 \pm 0.25$ \\
\hline
\end{tabular}

*Mean \pm SD

Table 2: Fall rates and use of psychotropic drugs in control and intervention facilities, before and after implementation of the intervention.

\begin{tabular}{|c|c|c|c|c|c|c|}
\hline & \multicolumn{2}{|c|}{ Control $(n=334)$} & \multicolumn{2}{|c|}{ Intervention $(\mathrm{n}=38 \mathrm{I})$} & \multicolumn{2}{|c|}{ Statistics } \\
\hline & Before & After & Before & After & $\mathrm{RR}^{*}$ & $95 \% \mathrm{Cl}$ \\
\hline \multicolumn{7}{|l|}{ Falls } \\
\hline Residents who fell in 3 months prior (\%) & 19.8 & 21.9 & 22.0 & 25.5 & 1.17 & $0.86-1.58$ \\
\hline \multicolumn{7}{|l|}{ Any psychotropics (\%) } \\
\hline Residents Prescribed & 63.8 & 68.1 & 70.0 & 69.9 & 0.89 & $0.69-1.15$ \\
\hline Administered Regularly & 45.2 & 48.8 & 40.2 & 40.4 & 0.93 & $0.82-1.05$ \\
\hline Marked PRN (\& not Reg.) & 12.2 & 8.8 & 23.1 & 16.3 & 1.48 & $0.79-2.77$ \\
\hline \multicolumn{7}{|l|}{ Benzodiazepines (\%) } \\
\hline Residents Prescribed Any Benzodiazepines & 43.7 & 43.7 & 45.4 & 39.1 & 0.89 & $0.69-1.15$ \\
\hline Residents Prescribed Long Acting Benzodiazepines & 13.8 & 12.9 & 10.9 & 8.1 & 0.76 & $0.56-1.03$ \\
\hline Residents Prescribed Short Acting Benzodiazepines & 35.3 & 34.1 & 37.7 & 32.3 & 0.95 & $0.70-1.28$ \\
\hline Any Administered Regularly & 32.0 & 32.0 & 28.1 & 24.7 & 0.87 & $0.69-1.09$ \\
\hline Any Marked PRN (\& not Reg.) & 8.8 & 7.8 & 17.2 & 13.0 & 1.31 & $0.62-2.73$ \\
\hline \multicolumn{7}{|l|}{ Antipsychotics (\%) } \\
\hline Residents Prescribed & 24.6 & 23.1 & 23.6 & 24.9 & 0.96 & $0.69-1.34$ \\
\hline Administered Regularly & 18.6 & 22.5 & 17.6 & 20.5 & 1.01 & $0.87-1.18$ \\
\hline Marked PRN (\& not Reg.) & 4.5 & 0.9 & 6.6 & 4.5 & 4.95 & $1.69-14.50$ \\
\hline
\end{tabular}

* Comparison of Intervention and Control groups post intervention after adjusting for pre-intervention results and statistically significant covariates. Notes: Percentages were calculated from residents present during both audits. The category "psychotropics" include residents prescribed any of benzodiazepines, antipyschotics or antidepressants. Marked PRN (\& not Reg.) refers to medications administered as the occasion arose but not regularly administered.

With the exception of one measure we were unable to detect statistically significant differences between the groups for the psychotropic drug use of the patients before or after the intervention. The exception was significantly greater use of "as required" antipsychotics in the intervention group compared with the control group after the pharmacy intervention $(\mathrm{RR}=4.95 ; 95 \% \mathrm{CI} 1.69-14.50)$ (Table 2).

There were no differences between groups for the recording of blood pressure or the percentages of patients with a recorded blood pressure of greater than 140/90 mm Hg. The percentage of residents on aspirin, percentage of resi- dents with atrial fibrillation and use of warfarin was similar between groups (Table 3).

\section{Discussion}

A systematic review of interventions designed to change physician's performance reported that outreach visits were an effective strategy [19]. Furthermore, the more educational methods are used the greater the chance of changing practice [19]. This randomised controlled trial used outreach visits incorporating audit, feedback and education. Despite the previous success of this approach in a residential care setting [1], we were unable to detect a 
Table 3: Blood pressure recording, patients at risk of stroke and use of aspirin and warfarin in control and intervention facilities, before and after implementation of the intervention.

\begin{tabular}{|c|c|c|c|c|c|c|c|}
\hline & \multicolumn{2}{|c|}{ Control } & \multicolumn{2}{|c|}{ Intervention } & \multicolumn{3}{|c|}{ Statistics } \\
\hline & Before & After & Before & After & $\mathrm{RR}^{*}$ & $P$ value & $95 \% \mathrm{Cl}$ \\
\hline BP Recorded in prior 3 months & 36.5 & 46.4 & 31.9 & 46.8 & 0.94 & 0.491 & $0.80-1.11$ \\
\hline BP recorded and Systolic BP > $140 \mathrm{~mm} \mathrm{Hg}{ }^{\ddagger}$ & 50.0 & 47.7 & 43.4 & 46.7 & 1.01 & 0.955 & $0.69-1.47$ \\
\hline $\mathrm{BP}$ recorded and Diastolic BP $>90 \mathrm{~mm} \mathrm{Hg} \ddagger$ & 5.7 & 4.5 & 4.4 & 0 & \# & & \\
\hline At Risk of Stroke & 59.0 & 65.0 & 54.9 & 57.7 & 0.65 & 0.096 & $0.39-1.08$ \\
\hline On Aspirin & 38.9 & 41.0 & 33.4 & 35.4 & 0.98 & 0.608 & $0.89-1.06$ \\
\hline Residents at risk of stroke and on Aspirin§ & 50.3 & 52.5 & 41.6 & 44.1 & 0.54 & 0.048 & $0.29-1.00$ \\
\hline Residents with Atrial Fibrillation recorded & 9.3 & 10.5 & 9.4 & 9.4 & 0.90 & 0.688 & $0.54-1.49$ \\
\hline $\begin{array}{l}\% \text { of Residents with Atrial Fibrillation } \\
\text { recorded on Warfarinll }\end{array}$ & 22.6 & 17.1 & 8.6 & 16.7 & 0.92 & 0.900 & $0.23-3.59$ \\
\hline
\end{tabular}

Analyses were adjusted for age, gender, level of care, dementia and baseline values. * Comparison of Intervention and Control groups post intervention after adjusting for pre-intervention results and statistically significant covariates. $\dagger$ Relative risk unable to be calculated due to insufficient numbers $\ddagger$ : Control $(n=122)$ and Intervention $(n=113) \S$ Control $(n=193)$ and Intervention $(n=2 \mid 2) \|:$ Control $(n=3 \mid)$ and Intervention $(n=36)$

change in the management of patients at risk of falls or stroke in the participating residential care facilities.

Some aspects of the trial design may have reduced the likelihood of an effect. The timeframe of the trial limited the level of involvement of the facilities and impacted on the sustainability of any changes. The rate of reporting of falls increased in both groups probably reflecting an increase in the awareness and documentation of falls and the rate of attrition of residents over the period of followup was higher than expected with $23 \%$ of residents moving or dying during the period of the trial. There were also difficulties with delivering the message in the residential care setting with a high turnover of staff. The attrition rate of staff was $13 \%$ in a six month period and a number of physicians attending the facilities had only one or two patients. Participating physicians cared for $59 \%$ of the residential care residents whose notes were audited and the omission of the other GPs may have diluted the overall effect. Many of these limitations may have impacted on the results found however we did conduct a satisfaction survey with residential care staff and focus groups with the facility staff and physicians which indicated that the detailing visits were important in increasing awareness, this is the subject of a separate manuscript. Both intervention and control facilities were audited at the beginning and end of the study and this scrutiny may have impacted on reporting and recording practices in both groups affecting the recording of outcomes eg falls.

This project highlighted many barriers to the successful implementation of evidence based clinical practice in residential care. Collection of data via case note audit required patient consent and for around $50 \%$ of residents this involved next of kin consent. Developing evidence based guidelines for managing patients in residential care was limited by the few trials including adults over 80 years of age. Furthermore, there were complexities in the development of best clinical management. An example of this was the recommended use of aspirin or warfarin to reduce stroke risk versus the potential for bleeding complications.

Structural issues in residential care may impact on the way that care is delivered. The decision to use many medications, particularly psychotropic medications, is influenced by a variety of people including the patient, residential care staff and the patient's next of kin as well as the physician. Carers, rather than nurses, are responsible for most of the day-to-day monitoring of residents behaviours and interventions focusing on professionals such as doctors and nurses may not be sufficient in this setting. In Australia current funding arrangements for residential care include few incentives for either physicians or residential care administration staff to improve clinical care and accreditation processes largely focus on accommodation issues rather than health care. General practitioners are under-supported due to an absence of medical advisory boards, a lack of audit, review and monitoring systems and communication difficulties between physicians, nursing staff and carers.

We were pleased, however, to find substantial interest in this trial from both the facilities and the physicians who visit them. This has resulted in commitment to a further project examining methods for enhancing communication within residential care facilities via case conferencing. This trial also resulted in the establishment of links between the hospital and residential care with future educational courses underway. 


\section{Conclusions}

This project found that while an outreach visit strategy was well received by physicians and facility staff involved it did not result in changes to the clinical management of residents. It highlighted the challenges of delivering evidence based care in a setting in which there is a paucity of well controlled trial evidence but where significant health outcomes can be attained. Residential care is a unique primary care setting. Interventions designed to change patient care require the inclusion of their usual physician and other individuals involved with the patient such as the residential care staff and next of kin. Furthermore, implementing evidence based practice in residential care setting represents a substantial challenge due to the lack of good quality evidence.

\section{Competing interests}

None declared.

\section{Author's contributions}

MC conceived of the study, participated in its design and coordination, and drafted the manuscript.

CW participated in the design and coordination of the study.

DR participated in the design and coordination of the study.

JH drafted the manuscript.

DW participated in the design and coordination of the study.

PF participated in the design and coordination of the study.

AE participated in the design of the study and performed the statistical analyses.

All authors read and critically reviewed drafts of the manuscript.

\section{Acknowledgements}

This research was supported by the National Health \& Medical Research Council Evidence Based Clinical Practice Research Program (Grant No 98/ 3|40I). We acknowledge the assistance of Rachel Wundke (RN) and Marisa Barbarioli.

\section{References}

I. Avorn J, Soumerai SB, Everitt DE, Ross-Degnan D, Beers MH, Sherman D, Salem-Schatz SR, Fields D: A randomized trial of a program to reduce the use of psychoactive drugs in nursing homes. New Engl J Med 1992, 327:168-173.

2. Tinetti ME: Factors associated with serious injury during falls by ambulatory nursing home residents. J Am Geriatr Soc 1987, 35:664-668.
3. Lord SR: Falls in the elderly: admissions, bed use, outcome and projections. Med J Aust 1990, 153: I I7-II8.

4. Province MA, Hadley EC, Hornbrook MC, Lipsitz LA, Phillip Miller J, Mulrow CD, Ory MG, Sattin RW, Tinetti ME, Wolf SL: The effects of exercise on falls in elderly patients: a preplanned metaanalysis of the FICSIT trials. JAMA 1995, 273:I34|-1347.

5. Rubenstein LZ, Robbins AS, Josephson KR, Schulman BL, Osterweil $D$ : The value of assessing falls in an elderly population. Ann Intern Med 1990, I I 3:308-316.

6. Ray WA, Taylor JA, Meador KG, Thapa PB, Brown AK, Kajihara HK, Davis C, Gideon P, Griffin MR: A randomised trial of a consultation service to reduce falls in nursing homes. JAMA I997, 278:557-662.

7. Mathers C, Vos T, Stevenson C: The burden of disease and injury in Australia. AlHW cat.no. PHE I7, Canberra: AlHW 1999.

8. Collins R, Peto R, MacMahon S, Hebert P, Fiebach NH, Eberlein KA, Godwin J, Qizilbash N, Taylor JO, Hennekens CH: Blood pressure, stroke and coronary heart disease: part 2 . Short term reductions in blood pressure: overview of randomized drug trials in their epidemiological context. Lancet 1990, 335:827-838.

9. Antiplatelet Trialists' Collaboration: Collaborative overview of randomized controlled trials of antiplatelet therapy-I: Prevention of death, myocardial infarction and stroke by prolonged antiplatelet therapy in various categories of patients. BrMed J 1994, 308:8I-106.

10. Gurwitz JH, Monett J, Rochon PA, Eckler MA Avorn J: Atrial fibrillation and stroke prevention with warfarin in the long-term care setting. Arch Intern Med 1997, I 57:978-984.

II. Soumerai SB: Principles and uses of academic detailing to improve the management of psychiatric disorders. Int J Psychiatry in Medicine 1998, 28:81-96.

12. Gillespie LD, Gillespie WJ, Cumming R, Lamb SE, Rowe BH: Interventions to reduce the incidence of falling in the elderly (Cochrane Review). In: The Cochrane Library Issue 3 Oxford: Update Software; 1998

13. South East Institute of Public Health (SEIPH): Guidelines for the prevention of falls in older people. Health Promotion Division of the Department of Health 1998.

14. Guidelines subcommittee: 1999 World Health Organization International Society of Hypertension guidelines for the management of hypertension. J Hypertens 1999, 17:151-183.

15. Collin C, Wade DT, Davies S, Horne V: The Barthel ADL Index: a reliability study. Int Disabil Stud 1998, 10:63.

16. Tinetti ME, Ginter SF: The Nursing Home Life-Space Diameter: a measure of extent and frequency of mobility among nursing home residents. Am Geriat Soc 1990, 38: I3 II-1315.

17. Ray WA, Taylor JA, Lichtenstein MJ, Meador KG: The Nursing Home Behavior Problem Scale. J Gerontol 1992, 47(I):M9-16.

18. StataCorp. Stata Statistical Software, Release 7.0. College Station, TX: Stata Corporation 200I.

19. Davis DA, Thomson MA, Oxman A, Haynes BR: Changing physician performance: a systematic review of the effect of continuing medical educational strategies. JAMA 1995, 274:700-705

\section{Pre-publication history}

The pre-publication history for this paper can be accessed here:

http://www.biomedcentral.com/1472-6963/4/6/prepub 